\title{
ARTE, POLITICA, CULTURA NEI GIARDINI DI VILLA MELZI D'ERIL A BELLAGIO. IL MONUMENTO A DANTE E BEATRICE DI GIOVANNI BATTISTA COMOLLI, 1810
}

\author{
Nota della s.c. ORNELLA SELVAFOLTA (*)
}

(Adunanza del 6 maggio 2021)

SunTO. - In occasione del duplice anniversario - settecento anni dalla morte di Dante Alighieri e duecento dalla morte di Napoleone Bonaparte - la Memoria affronta il tema del monumento a Dante e Beatrice scolpito dall'artista neoclassico Giovanni Battista Comolli nel 1810. Committente fu Francesco Melzi d'Eril, Vicepresidente della Repubblica Italiana (1802-1804), poi Gran Cancelliere Guardasigilli del Regno d'Italia (1805-1814). Situato nei giardini di villa Melzi d'Eril a Bellagio sul lago di Como, il monumento rappresenta l'incontro di Dante e Beatrice nel Canto XVIII del Paradiso, quando la donna consola il poeta della profezia dell'esilio annunciata dall'avo Cacciaguida. Il saggio mette in luce come la scelta del soggetto sottintenda da parte del committente il desiderio di celebrare il poeta e la sua opera quali espressioni di identità e valori italiani, nel momento in cui la svolta autoritaria di Napoleone aveva deluso le sue aspirazioni per una maggiore indipendenza del paese. La scultura presenta inoltre alcune novità dal punto di vista artistico e, nell'insieme, può essere considerata un esempio precoce di monumento specificatamente dedicato a Dante e alla Commedia, in anticipazione della fortuna artistica che la figura del poeta avrà nei decenni seguenti del XIX secolo.

$$
* * *
$$

AвSTRACT. - On the occasion of the double anniversary - the seven hundred years since the death of Dante Alighieri and the two hundred years since the death of Napoleon Bonaparte - the essay deals with the monument of Dante and Beatrice

(*) Politecnico di Milano, Italy. E-mail: ornella.selvafolta@polimi.it 
sculpted by the neoclassical artist Giovanni Battista Comolli in 1810. His client was Francesco Melzi d'Eril, former Vice President of the Italian Republic (1802-1804), then Grand Chancellor Keeper of Seals of the Kingdom of Italy (1805-1814). Located in the gardens of the Villa Melzi d'Eril in Bellagio on Lake Como, the monument features the meeting of Dante and Beatrice in Canto XVIII of Paradiso, when the woman soothes the poet for the prophecy of exile announced by his ancestor Cacciaguida. The paper highlights how this subject implies on the part of the client the desire to celebrate the poet and his work as expressions of Italian values, at a time when Napoleon's authoritarian turn had disappointed his aspirations for the country's greater independence. The sculpture also marks some artistic novelties and, as a whole, can be considered an early example of a monument specifically designed around Dante and his Commedia, anticipating the artistic success of the poet in the following decades of the Nineteenth century.

\section{PREMESSA}

Al di là delle occasioni puramente celebrative, gli anniversari, offrono l'opportunità di ampliare le indagini storico-critiche, di riportare alla luce fonti poco note o poco considerate, di affrontare argomenti altrimenti trascurati, e di collegare, ove è possibile l'eredità del passato con le circostanze dell'attualità e i significati che essa può assumere nel presente. La duplice ricorrenza, nel 2021, dei settecento anni dalla morte di Dante Alighieri e dei duecento da quella di Napoleone Bonaparte, ha suggerito la riflessione su un tema storico-artistico che, oltre a comprendere entrambi i protagonisti, intercetta le vite di artisti, committenti e istituzioni di grande rilievo nell'Italia del primo Ottocento, e, contemporaneamente, evidenzia l'esistenza di tendenze artistiche e istanze politico-ideologiche variamente orientate verso una crescente consapevolezza dell'identità nazionale ${ }^{1}$.

L'opera in questione è il monumento intitolato Dante e Beatrice, realizzato tra il 1809 e il 1810 dallo scultore neoclassico Giovanni Battista Comolli per Francesco Melzi d'Eril, duca di Lodi e figura di primo piano dell'Italia napoleonica. Collocato nel 1814 nei giardini della sua villa appena costruita a Bellagio, sul lago di Como, il gruppo rappresenta l'incontro di Dante con Beatrice nel Canto XVIII del

1 Cfr., proprio in riferimento alle figure di Dante e Napoleone, la mostra e il contributo recente di D'Adda - Onger (a cura di) 2021. 
Paradiso, quando la donna consola il poeta della profezia dell'esilio trasmessagli dall'avo Cacciaguida. Un soggetto inusuale e certamente non casuale che, come si vedrà, proietta la memoria del sommo poeta nell' "attualità napoleonica" attraverso un sottile gioco di allusioni e rimandi simbolici che dalla trama letteraria si trasmettono alla rappresentazione artistica.

Da quest'ultimo punto di vista la scultura presenta alcune novità e, nell'insieme, può essere considerata uno dei primi monumenti espressamente dedicati alla figura di Dante e alla sua creazione letteraria, pensato come opera a sé stante, che si erge autonomamente nello spazio, indipendente da architetture, ripartizioni costruttive e decorative, da luoghi e funzioni commemorative. E' quindi un esempio precoce della fortuna monumentale incontrata dal poeta soprattutto nella stagione postrisorgimentale, quando numerose piazze d'Italia si arricchiranno della sua effigie ${ }^{2}$.

Tuttora esistente nei giardini di Bellagio, il monumento ne ha costituito e ne costituisce una presenza caratterizzante, non a caso ricordata in tutte le guide di viaggio, descrizioni e memorie che, nel corso dei decenni, hanno accompagnato i passi dei visitatori, fino a diventare un vero landmark che, oggi come allora, merita di essere evidenziato nei suoi contenuti di arte e di storia.

\section{LA VILLA E I GIARDINI DI FRANCESCO MELZI D'ERIL A BELlaGIO}

Alcune coordinate essenziali al contesto sono necessarie: la villa e i giardini di Bellagio furono realizzati per Francesco Melzi d'Eril tra il 1809 e il 1813, in un luogo considerato tra i più belli del Lario per il suo situarsi a cavallo dei due rami lacustri e per godere, quindi, di particolare vantaggi paesaggistici ${ }^{3}$. Responsabile del progetto architettonico e decorativo della villa e della cappella gentilizia, fu Giocondo Albertolli, fondatore e professore della Scuola di Ornato all'Accademia di Belle Arti di Brera, già collaboratore di Giuseppe Piermarini nelle sue più famose e

2 Nella ricca bibliografia su questo tema, cfr. Tobia 1997, (http://www.persee.fr/doc/mefr_1123-9891_1997_num_109_1_4479)

3 Sulla villa e i giardini Melzi d'Eril a Bellagio rimando a Selvafolta 2012. Altri contributi più specifici saranno menzionati in corrispondenza del testo. 
prestigiose realizzazioni, quali ad esempio, palazzo Reale di Milano o la Villa Reale di Monza4.

Albertolli influì in modo permanente sulle vicende dell'arte, del décor e del gusto neoclassico nel lungo protrarsi tra Sette e Ottocento, grazie alla maturazione di un linguaggio decorativo che sapeva integrare architettura e ornato, dove le forme classiche si combinavano con rielaborazioni rinascimentali. L'incarico affidatogli da Melzi d'Eril a Bellagio rappresentò per lui un traguardo fondamentale, perché fu l'unico nella sua lunga attività, a includere architettura e decorazione con un ruolo che andava oltre il semplice progetto, comprendendo la supervisione e il controllo sul processo esecutivo e sulla qualità dei lavori, ivi compresi gli interventi degli artisti (Fig. 1).

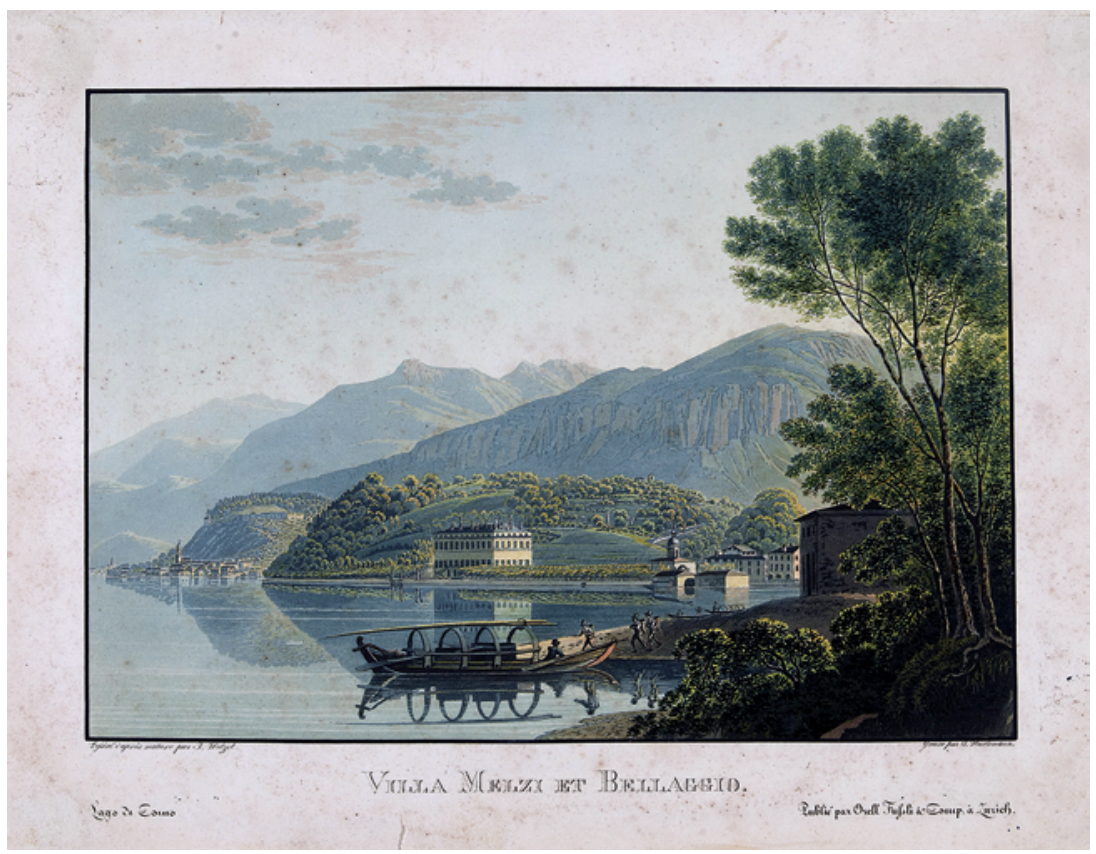

Fig. 1 - Veduta di Villa Melzi e Bellagio di Jacob Wetzel, Zurigo 1822. Bellagio, Museo Lodovico Gallarati Scotti.

4 Nella bibliografia su Giocondo Albertolli (1743-1839) cfr. Colle - Mazzocca 2005 e, da ultimo, Agliati - Cordera - Ricci (a cura di) 2019. Per lo scenario artistico del neoclassicismo milanese e lombardo cfr. Mazzocca - Morandotti - Colle 2001. 
Responsabili del disegno e della realizzazione dei giardini furono l'architetto Luigi Canonica e l'agronomo Luigi Villoresi: il primo di origine ticinese ma formatosi all'Accademia di Brera, fu «architetto di stato», progettista ufficiale della Milano francese e, contemporaneamente, fu attivo per un'elevata committenza privata; ${ }^{5}$ il secondo fu tra $\mathrm{i}$ più apprezzati botanici della prima metà dell'Ottocento, capace di coniugare il sapere scientifico con la pratica delle coltivazioni e una perfetta padronanza dell'arte dei giardini. ${ }^{6}$ Insieme avevano già lavorato alla riforma del parco della Reggia di Monza riprogettato e ampliato per volere di Eugenio de Beauharnais che, nel 1805, ne era entrato in possesso quale Vicerè del nuovo Regno d'Italia.

Sulle sponde del lago operarono quindi alcuni tra i più qualificati professionisti del periodo, attivi per un committente, come Francesco Melzi d'Eril che, a sua volta, si distingueva per la qualità dell'agire, per l'apertura culturale, il senso politico e la passione civile. Sulla sua figura esistono numerosi e rilevanti studi'; si possono qui solo evidenziare, e in estrema sintesi, alcuni aspetti che più hanno attinenza con la villa di Bellagio e con il significato del monumento a Dante e Beatrice.

Di antica famiglia aristocratica, Melzi aveva compiuto gli studi umanisti e scientifici a Milano, e aveva proficuamente arricchito la sua educazione con numerosi viaggi in Europa (Inghilterra, Francia e Spagna) che gli aprirono un orizzonte internazionale di pensieri ed esperienze. Partecipe della cultura tardo illuminista, le sue aspirazioni erano indirizzate a uno sviluppo graduale delle istituzioni in senso liberale, che non scardinasse la struttura sociale e i diritti dell'aristocrazia. Nel 1796 aveva accolto con favore l'arrivo in Italia di Napoleone che, personalmente, ne apprezzò sempre la serietà e l'impegno anche nei periodi segnati da una minore "sintonia" reciproca.

Nel 1802 egli fu nominato Vicepresidente della Repubblica Italiana presieduta da Napoleone, trovandosi a reggere la complessa organizzazione politica e amministrativa di uno stato in formazione,

5 Per Luigi Canonica (1762-1844), cfr. Tedeschi - Repishti (a cura di) 2011.

6 Per Luigi Villloresi (1779-1823), cfr. Pellissetti 2009, 301-303.

7 Per i giardini della villa Reale di Monza rimando, tra i numerosi studi di Marina Rosa, a: Rosa (a cura di) 2009 e, per il periodo francese, Rosa 2014, 131sgg.

8 Per Francesco Melzi d'Eril (1753-1816) figlio di Gaspare e della gentildonna spagnola Maria Teresa d'Heryl, da cui prese il secondo cognome, cfr. nell'ampia bibliografia: Del Bianco 2002; Capra 2009, ad vocem. 
bisognoso di riforme in pressoché tutti i settori della vita civile e militare: dalla creazione di un esercito nazionale al risanamento delle finanze, dalla riforma dell'istruzione a quella del sistema giudiziario' (Fig. 2).

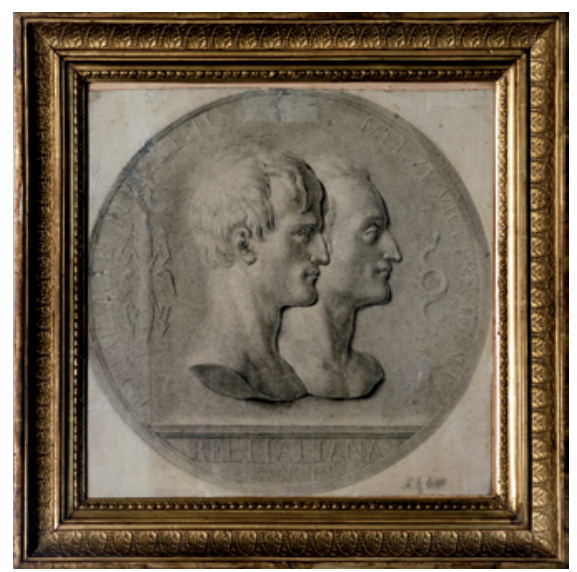

Fig. 2 - Disegno di Giuseppe Bossi per una medaglia con i profili

di Napoleone Bonaparte e di Francesco Melzi d'Eril, negli anni della Repubblica Italiana 1802-1804.

Bellagio, Museo Lodovico Gallarati Scotti.

Degno di nota fu anche l'interesse per le arti, che rientravano nel suo orizzonte culturale, non tanto o non solo come attitudine personale o come desiderio di decoro per le sue dimore, quanto per la convinzione, altamente civile, che fosse una sorta di dovere morale promuovere un patrimonio di creatività, esperienza e lavoro proprio alla cultura italiana, di cui anche la villa di Bellagio e il monumento a Dante e Beatrice sono testimonianza. La sua fu quindi un'attività a tutto campo, costellata di risultati alterni, ma sempre improntata a uno spiccato sentimento dello stato e, soprattutto, dello "stato italiano", per il quale Melzi d'Eril auspicava un ruolo di maggiore autonomia nello scenario politico.

Il suo percorso fu segnato dalla progressiva escalation al potere di Napoleone, Presidente della Repubblica Italiana nel 1802, Imperatore

9 Per la Repubblica Italiana, cfr. Capra - Della Peruta - Mazzocca 2002. In generale sul panorama istituzionale e culturale del periodo: Brambilla - Capra - Scotti (a cura di) 2008. 
nel 1804, Re d'Italia nel 1805: fu, come è noto, un "cambio di passo", una svolta in senso autoritario che mise fine alle esperienze repubblicane e, contestualmente, ridimensionò le aspirazioni di Melzi. Nel nuovo ordinamento del Regno egli ricoprì cariche di prestigio come membro del Consiglio di Stato, Grand'Ufficiale della Corona e Cancelliere Guardasigilli; affiancò il giovane Viceré Eugenio de Beauharnais per assisterlo nelle più complesse decisioni di governo e promuovere, per quanto possibile, gli interessi italiani, ma vide irrimediabilmente ridotto il suo ruolo politico e gradualmente vanificati i suoi progetti.

Nel 1807 Napoleone lo nominò «duca di Lodi», conferendogli un titolo senza feudi che, tuttavia, poteva essere trasmesso agli eredi e garantiva un appannaggio di 200.000 lire annue, in ragione dei servizi «resi all'amministrazione pubblica» e in ricordo dell'incontro avvenuto più di dieci anni prima, a Lodi, dopo la battaglia vittoriosa che aveva aperto alle truppe francesi l'ingresso a Milano ${ }^{10}$. L'autorità e i vantaggi economici che ne derivarono consentirono a Melzi d'Eril, di realizzare la villa e i giardini di Bellagio ove trascorrere i periodi meno oberati di impegni, in un clima adatto alla sua salute cagionevole come a una crescente disaffezione dalla politica.

Tra il 1807 e il 1808 il duca iniziò quindi ad acquistare diversi terreni sul lago di Como, di fronte alla costa della Tremezzina, confinanti col porticciolo di Loppia ed estesi verso il borgo di Bellagio, fino a disporre, nell'arco di circa un anno, di una proprietà sufficientemente ampia da poter essere attorniata da un grande giardino.

Canonica e Villoresi sistemarono a tale scopo una porzione di terreno di circa 10 ettari, adottando tra i primi sul lago di Como i principi del giardino paesaggistico diffusi a inizio Ottocento in Lombardia soprattutto grazie alla circolazione del trattato di Ercole Silva, Dell'arte de' giardini inglesi $i^{11}$. In armonia con il sito lacustre essi plasmarono un terreno di forma irregolare, traendo vantaggio dalla varietà dei rilievi altimetrici, dal diverso andamento dei percorsi, dalle alternanze tra

10 Cfr. Bascapè - Dal Piazzo (a cura di) 1983, 804-806; cfr. N. 276 Reale lettera 1807, 1225-1226, dove si legge: «fu il primo Italiano che ci portò sul campo di battaglia a Lodi, le chiavi e i voti della nostra buona città di Milano. Noi abbiamo risoluto di conferirgli il titolo di duca di lodi, per essere portato da lui e da' suoi eredi maschi, tanto legittimi e naturali che adottivi, per ordine di primogenitura [...]».

11 Cfr. Selvafolta 2020, 90-95. Per il contributo di Ercole Silva (1756-1840) cfr. Silva 1813. 
parti collinose e parti in piano, tra spazi aperti e spazi raccolti, tra l'infittirsi e il diradarsi della vegetazione nella disposizione "libera" della componente botanica.

Contava nel progetto il modo in cui il giardino si inseriva nel luogo, abbellendolo senza cancellarne la "naturalità": tanto più auspicabile a Bellagio ubicato su un promontorio al centro del lago e a spartiacque tra i suoi due rami, in una "postura" particolarmente felice per la varietà orografica, la vivacità delle luci e dei colori, la diversità dei venti e dei cieli, per i risalti multipli dei pendii e delle sponde. In questo contesto la villa del duca si segnalava anche per la specifica qualità di essere sorta completamente ex-novo in base a un progetto unitario che, fin dall'inizio, aveva riguardato l'architettura e le arti, il territorio e il paesaggio, la natura e il giardino.

A differenza di altri esempi lariani, altrettanto pregevoli e famosi nel primo Ottocento, essa non era infatti il risultato di diverse acquisizioni nel tempo, né di trasformazioni di insediamenti esistenti e neppure il portato di differenti inclinazioni di gusto, bensì l'esito di un intervento pensato nella sua totalità dove tutte le parti erano trattate in coerenza, stabilendo una trama di connessioni e rimandi che, a sua volta, poteva essere considerata tra le precipue virtù dei "moderni" giardini paesaggistici.

Il che implicava una profonda intesa tra il committente e i progettisti, all'origine di un disegno senza sbavature dove tutto si tiene secondo un equilibrio che Cesare Cantù nel 1847 definiva con calzante espressione «di sostanza» più che di «apparenza» ${ }^{12}$. Vi rientrava anche la presenza di manufatti scultorei: una prerogativa specifica di villa Melzi d'Eril, che seppure "poco praticata" dai principi del giardino paesaggistico, nel primo Ottocento e nell'Italia napoleonica viveva un'accentuata fortuna in

12 C. Cantù 1847, 60. L'armonia d'insieme del complesso di Bellagio è ancora oggi percepibile, grazie anche al fatto che la proprietà è sempre rimasta nell'ambito della stessa famiglia la quale, pur con gli inevitabili cambiamenti culturali e funzionali nei due secoli trascorsi dalla realizzazione, ha operato nel segno di una sostanziale continuità e rispetto delle scelte originarie. Ricordo in sintesi che alla morte del duca Francesco nel 1816, la proprietà passò al nipote Giovanni Francesco, indi al figlio di questi Lodovico, successivamente, alla moglie Joséphine Barbò, la cui figlia, Luisa Melzi d'Eril, sposò nel 1878 il principe Giancarlo Gallarati Scotti. La villa e i giardini furono quindi ereditati dal primogenito, duca Tommaso Gallarati Scotti, trasmettendosi poi ai discendenti della famiglia in linea maschile. 
concomitanza con la temperie artistica del Neoclassicismo e con le strategie di celebrazione e propaganda politica attraverso le immagini ${ }^{13}$.

A Bellagio la scultura ebbe quindi funzione di abbellimento, ma anche di testimonianza ideologica e culturale, risultando variamente calata nell'attualità del tempo, sia per la scelta dei soggetti, sia per quella degli artisti, tra i quali risalta particolarmente la figura di Giovanni Battista Comolli: l'autore del monumento a Dante e Beatrice (Fig. 3).

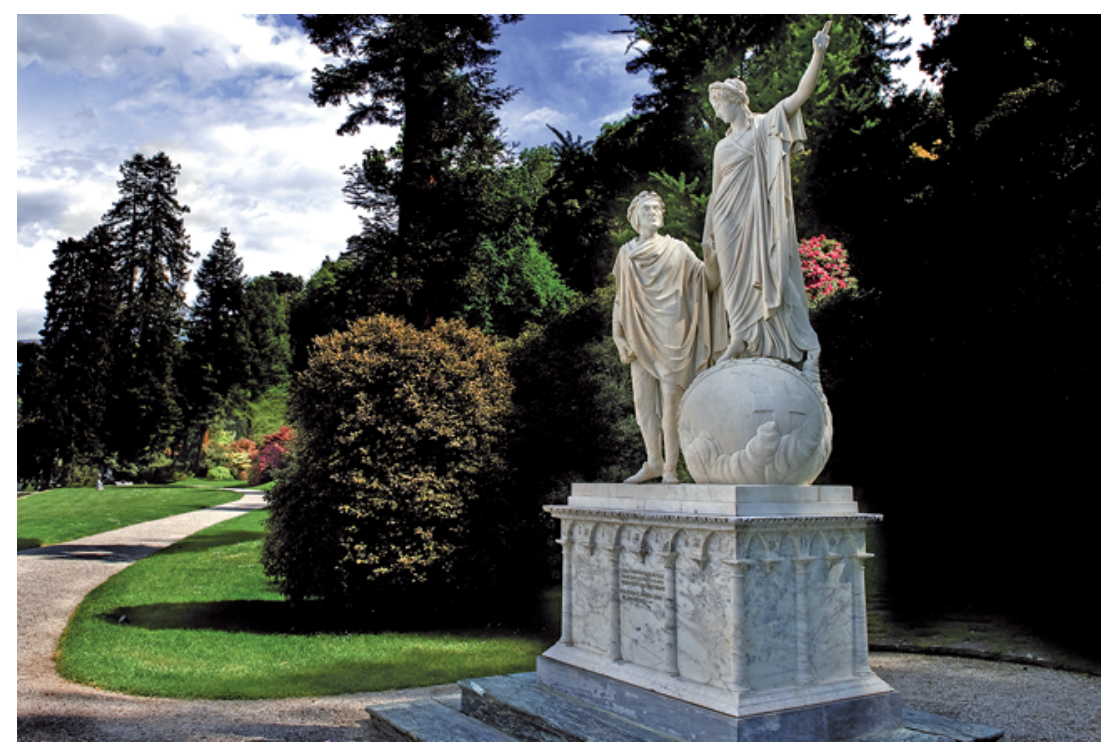

Fig. 3 - Il monumento a Dante e Beatrice nei giardini di villa Melzi d'Eril a Bellagio. Scultore: Giovanni Battista Comolli, 1810. Foto: Franco Papetti.

3. Giovanni BatTista Comolli Scultore (1775-1830). TRACCE ALL'ISTITUTO LOMBARDO ACCADEMIA DI SCIENZE E LETTERE

Nato nel 1775 a Valenza Po, formatosi nelle Accademie di Milano e di Roma, Comolli fu artista engagè, giacobino e repubblica-

13 Rimando al contributo. ormai "classico". di Hubert 1964. Cfr., più in generale, anche in rapporto alle altre forme di rappresentazione artistica, Mazzocca 2021. 
no, convinto filofrancese ${ }^{14}$ La sua vicenda biografica intreccia professione e fede politica con esiti non sempre facili, segnati da successi e insuccessi, consensi e opposizioni, mantenendo la sua produzione su un piano di indiscusso talento e qualità, ma camminando sul filo incerto degli eventi in continua trasformazione, quasi mai con una posizione o una residenza stabile, nell'alternarsi tra Roma e Torino, Grenoble e Parigi, Carrara, Londra, Milano...

Comolli fu autore di progetti monumentali secondo il registro epico che era proprio a quei tempi, ma fu specialmente apprezzato per i numerosi ritratti di personaggi pubblici e privati, che costituirono la parte più significativa e remunerativa della sua opera, procurandogli riconoscimenti e lavoro su scala internazionale, in parallelo con i molti itinerari della sua vita. Nei busti l'artista sapeva esprimere «il carattere stesso del personaggio», si sottolineava, per evidenziarne la capacità di associare la rappresentazione fedele dei tratti del volto con l'espressione del carattere e «l'immagine dell'animo» ${ }^{15}$.

Durante gli anni del suo soggiorno a Milano alla fine del primo decennio dell'Ottocento, egli lasciò tracce anche nella vita dell'Istituto Lombardo (allora Regio Istituto Italiano) proponendo nel 1812 all'allora Presidente Giovanni Paradisi, un busto di Napoleone Imperatore ${ }^{16}$. Definita «colossale» per le dimensioni maggiori del vero, la scultura, tuttora patrimonio dell'Istituto, consente di aprire una breve parentesi documentaria in base alle testimonianze dell'Archivio storico ${ }^{17}$. Nella lettera, datata 4 luglio 1812, Comolli prospettava l'opportunità per l'istituzione di possedere un ritratto di Napoleone, eseguito con «quell'amore che ispira naturalmente un Sovrano che tanto protegge le Belle Arti» e che, a suo avviso, sarebbe stato particolarmente adatto a «decorare la Sala delle Pubbliche Sedute» nel palazzo dell'Accademia di Belle Arti di Brera ${ }^{18}$ (Fig. 4).

14 Per Giovanni Battista Comolli (1775-1830) cfr. in prima istanza Kannès 1982, ad vocem; inoltre: Nicodemi 1915, 68-72; Grano (a cura di) 1990; Selvafolta 2010, 49-95; Grandesso 2013, 71-88.

15 Citazione da Giambattista Comolli 1831, 139.

16 Se ne ha notizia in Della Peruta, 2007, 113-114, 164.

17 Ringrazio la dottoressa Rita Pezzola, Cancelliere dell'Istituto, per la preziosa collaborazione nella ricerca delle fonti d'archivio riguardanti il busto di Napoleone Imperatore di G.B. Comolli.

18 Archivio dell'Istituto Lombardo Accademia di Scienze e Lettere, Milano (d'ora in poi ILASL), Titolo IV, Cart.37: Lettera di Giovanni Battista Comolli al Presidente dell'Istituto, 4 luglio 1812. 


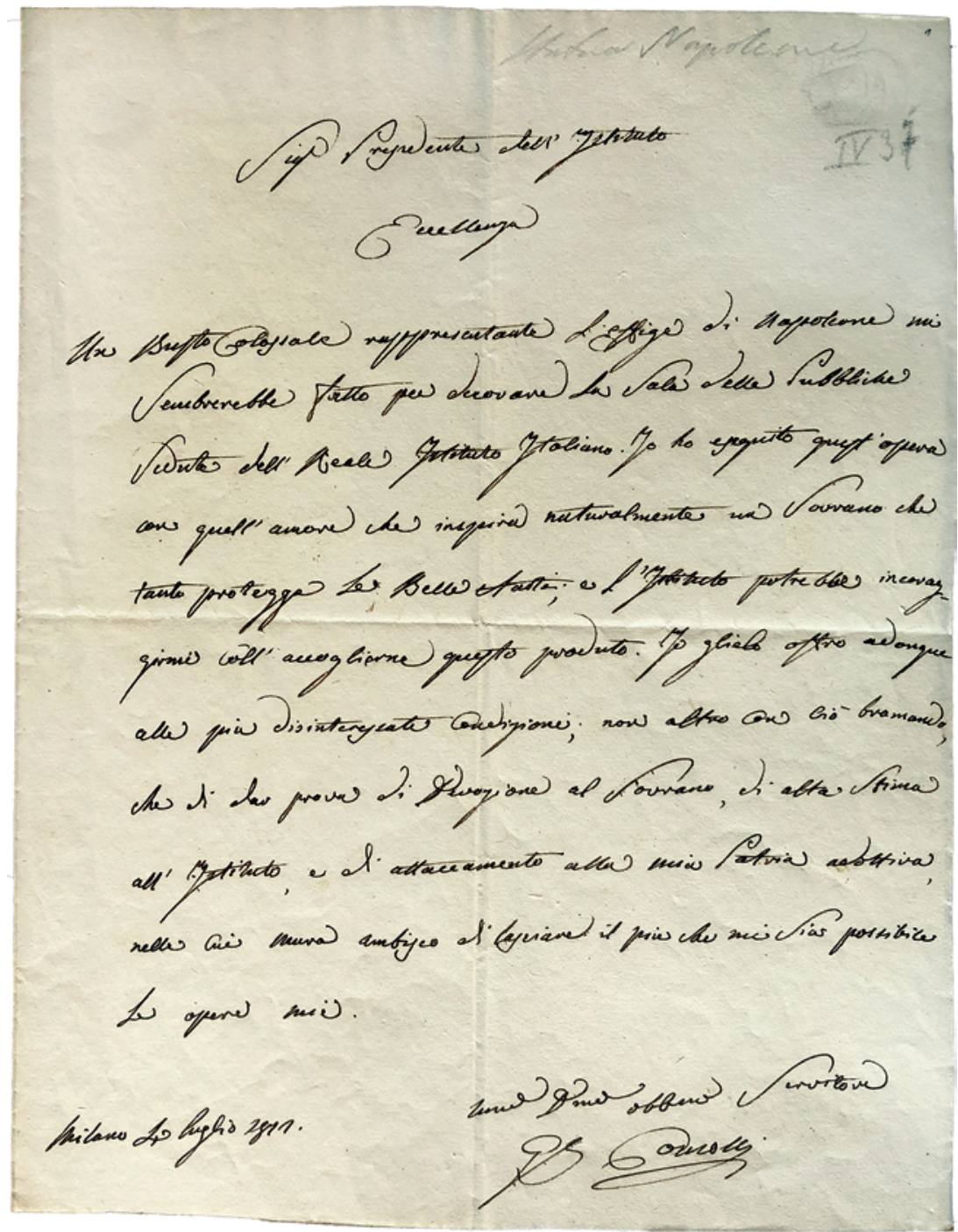

Fig. 4 - Lettera dello scultore Giovanni Battista Comolli al presidente dell'Istituto Lombardo in cui propone di acquisire un "busto colossale" di Napoleone Imperatore, Milano 4 luglio 1812.

Milano, Archivio dell'Istituto Lombardo Accademia di Scienze e Lettere (ILASL). 
Non vi sono indicazioni precise sulla sua realizzazione, ma si capisce che si tratta di un'opera già realizzata, forse durante i soggiorni dello scultore a Carrara tra il 1809 e il 1811 dove, come si vedrà, egli era impegnato a lavorare soprattutto per Francesco Melzi d'Eril e per il monumento a Dante e Beatrice. Sappiamo del resto come siano "innumerevoli" i busti di Napoleone scolpiti in quegli anni, con fattezze più o meno idealizzate e, per lo più, derivate dal famoso ritratto di AntoineDenis Chaudet di inizio secolo e Comolli non fa eccezione.

Egli è autore di almeno tre esemplari, di dimensioni «colossali», oggi conservati nelle raccolte milanesi: Napoleone Re d'Italia della Galleria d'Arte Moderna, Napoleone Imperatore e Re d'Italia del Museo del Risorgimento e quello dell'Istituto lombardo ${ }^{19}$. Quest'ultimo si distingue per essere inequivocabilmente dedicato a Napoleone Imperatore: è infatti l'unico privo della Corona Ferrea e che ha espliciti rimandi all'iconografia antica del trionfatore sui campi di battaglia, con il capo cinto dal serto di alloro, una spalla drappeggiata con la clamide, l'altra vestita dell'armatura (Fig. 5).

La lettera redatta da Comolli, con con il pathos che spesso contrassegna la sua scrittura, segnalava che la sua era un'offerta alle «più disinteressate condizioni, non altro con ciò bramando, che di dar prova di devozione al Sovrano, di alta stima all'Istituto, e di attaccamento alla mia Patria adottiva [la città di Milano] nelle cui mura ambisco di lasciare il più che mi sia possibile le opere mie» ${ }^{20}$. Nella missiva non si parla di costi, ma la situazione economica dello scultore, quasi mai florida, fa ipotizzare che offrire non significasse regalare. Il 4 febbraio 1813, Il verbale dell'Adunanza della Commissione di Amministrazione valutava infatti che, assolte le spese già programmate e tenuto conto delle previsioni di introiti dell'Istituto, si potesse ormai «ultimare il contratto del Busto di Sua Maestà offerto dallo scultore Comolli», tenuto fino ad allora in sospeso «per la mancanza di un fondo sufficiente» ${ }^{21}$.

Si deduce inoltre che l'opera era già stata sottoposta al giudizio di tre esperti artisti come il pittore Giuseppe Bossi, l'architetto Luigi Cagnola e l'incisore Giuseppe Longhi, membri della «Commissione per

19 Cfr. il sito Lombardia Beni Culturali, http://www.lombardiabeniculturali.it/ opere-arte/schede/2d070-00109/

20 ILASL, Titolo IV, Cart. 37, Lettera del 4 luglio 1812.

21 ILASL, Titolo V. Cart.1, Verbale dell'Adunanza 4 febbraio 1813. 
sovraintendere a ciò che occorre per la fabbrica e gli ornamenti delle Sale dell'Istituto» ${ }^{22}$.

La settimana successiva, l'11 febbraio 1813, dietro altre assicurazioni di qualità da parte di Bossi, nonostante alcune macchie nel marmo statuario che diminuivano il valore dell'opera, la questione si risolse acquistando, per la somma piuttosto considerevole di «90 Luigi» (equivalenti a circa 2175 lire) il busto dell'Imperatore unitamente al basamento in marmo grigio Bardiglio, decorato con bassorilievi allegorici di grifoni, corone di alloro, faci diritte accese e festoni, simboli della vigilanza, della potenza, della gloria ${ }^{23}$.

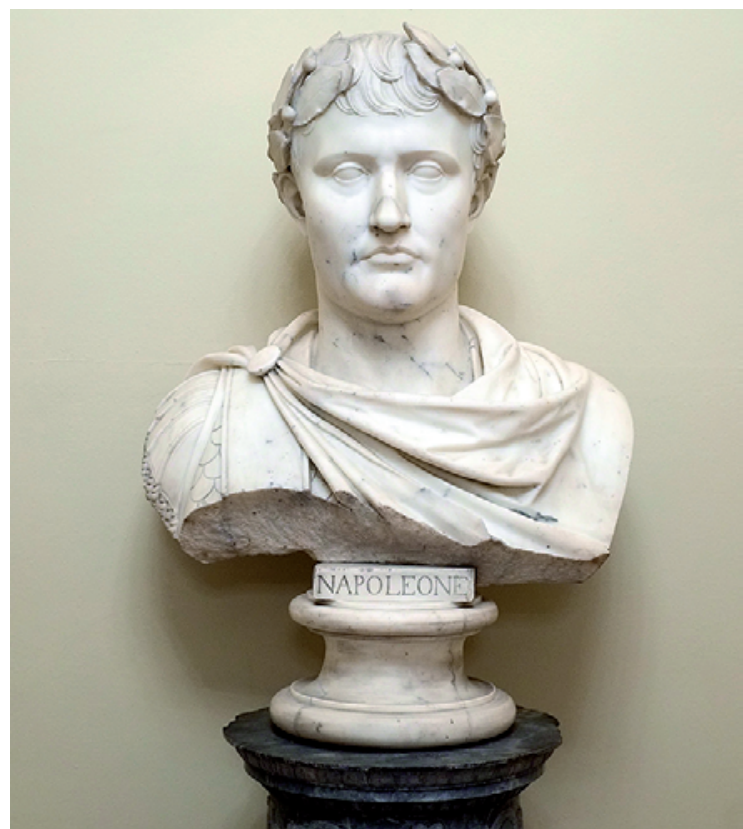

Fig. 5 - Il busto colossale di Napoleone Imperatore dello scultore

Giovanni Battista Comolli all'Istituto Lombardo Accademia di Scienze e Lettere. Milano, Sala delle Adunanze all'Accademia di belle Arti di Brera. Foto: Alberto Baroni.

22 ILASL., Titolo V, Cart. 1, Verbale dell'Adunanza 11 febbraio 1813.

23 La somma di 90 Luigi equivaleva a circa 2175 lire italiane. Devo l'informazione alla gentilezza e competenza del numismatico Davide Mauri che ha risolto questo non facile quesito e che desidero qui ringraziare. 
Il «busto colossale» dell'imperatore fu ceduto dallo scultore e acquisito dall'Istituto praticamente allo scadere del tempo napoleonico, diventando per entrambi una sorta di ultima possibilità, in parte già in controtendenza, di onorare il conquistatore. Collocato in piena evidenza nella Sala delle Adunanze, dovette infatti essere rimosso in base alle disposizioni impartite dagli Austriaci di eliminare dall'Istituto i simboli e i ricordi del governo precedente.

Nel 1816 il busto di Comolli fu riposto in «luogo appartato» ${ }^{24}$, mentre lo stesso scultore preferì lasciare Milano (Francesco Melzi d'Eril era morto all'inizio dell'anno) e riparare altrove in cerca di sicurezza e di nuove commesse. Soggiornò a Londra dal 1816 al 1820 dove lasciò opere pregevoli tra ritratti, sculture allegoriche, decorazioni e studi dall'antico; fece quindi ritorno a Milano e intraprese altri percorsi artistici e politici con alterne fortune (nel $1822 \mathrm{fu}$ in carcere per circa un anno con l'accusa di avere cospirato contro l'Austria $)^{25}$, lavorando anche per i nuovi dominatori, seppure mai immemore del suo ruolo di artista impegnato e del suo passato di fede francese.

\section{Comolli e Melzi D’ERIL, ARTista e COMmitTENTE. IL MONUMENTO A DANTE E BEATRICE}

Determinante nella vita professionale di Comolli fu Francesco Melzi d'Eril che aveva conosciuto lo scultore negli anni della Repubblica Cisalpina e aveva apprezzato un suo busto di Napoleone realizzato nel $1801^{26}$. Il loro rapporto si era poi consolidato nel periodo della Repubblica Italiana quando Comolli era stato incaricato di scolpire il busto ufficiale di Melzi Vicepresidente. La sua è un'opera "ispirata", che ritrae un personaggio dal volto idealizzato e con bei panneggi all'antica, e che, come nella migliore produzione dello scultore, aveva

24 Della Peruta 2007, 164. Il «busto colossale» di Napoleone è oggi conservato nella Sala delle Adunanze dell'Istituto Lombardo Accademia di Scienze e Lettere all'Accademia di Brera.

25 Kannès 1987, ad vocem: Comolli fu imprigionato il 3 aprile 1822 con «l'accusa di alto tradimento» per i suoi contatti con Federico Confalonieri e altre personalità, note per le loro posizioni antiaustriache, e per la sua, più presunta che effettiva, partecipazione ai moti del 1821. Fu rilasciato nel 1823 in assenza di prove concrete.

26 Kannès 1987, ad vocem; Selvafolta 2003. 
saputo individuare i tratti caratteristici del modello riprendendone le fattezze come veicolo di espressione morale. In quanto tale si era meritata un articolo elogiativo sul «Nuovo Giornale dei Letterati» corredato di alcuni «versi sciolti», un po' ampollosi, di Celestino Masucco, professore di Poesia all'Università di Genova, dove Comolli, era considerato artista «valoroso» e di «caldo ingegno» che non solo aveva ritratto «al vivo» il Vicepresidente, ma era anche riuscito a infondergli elevatezza e dignità di pensiero ${ }^{27}$ (Fig. 6).

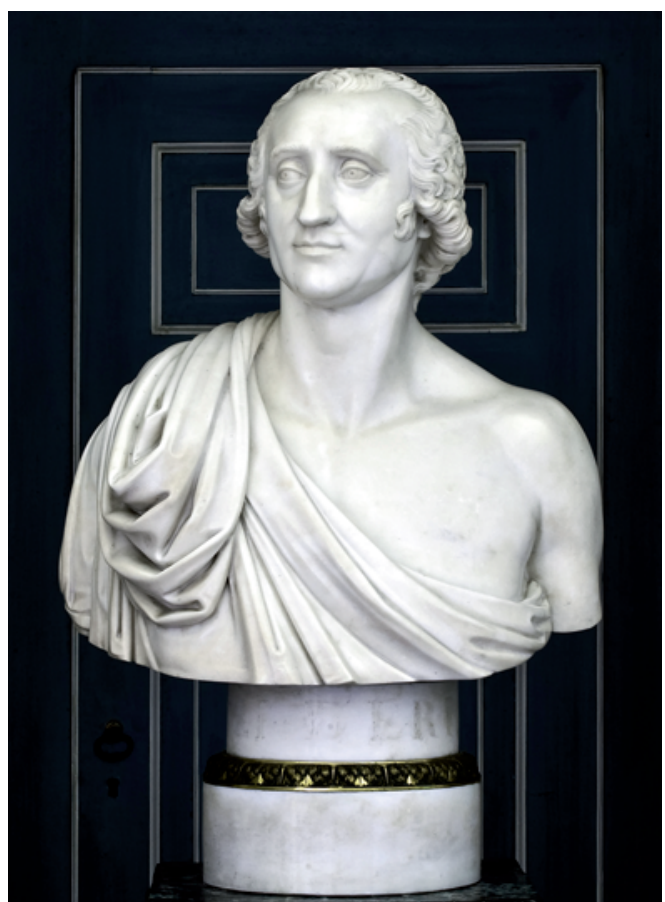

Fig. 6 - Busto di Francesco Melzi d'Eril vicepresidente della Repubblica Italiana. Scultore: Giovanni Battista Comolli, 1803. Collezione privata.

Foto: Franco Papetti.

27 Cfr. Busto del Vice-Presidente 1804, 130-134. Commissionato dal Governo della Repubblica, il ritratto, originariamente destinato alle «Sale del Palazzo Nazionale in Milano» (Palazzo Reale), è oggi conservato in Villa Melzi d'Eril a Bellagio. 
Da questi anni Comolli ebbe un rapporto privilegiato con Melzi d'Eril, intensificatosi nel corso del tempo fino a diventare quasi esclusivo nel periodo in cui si stava progettando e costruendo il complesso di Bellagio. A partire dal 1808 egli realizzò numerose opere, diverse per tipologia, impegno artistico, valore simbolico e contenuto tecnico, passando dai busti-ritratti al gruppo monumentale, dalla scultura sacra ai «lavori di ornato» e di arredo. Fu quindi scultore versatile e disponibile, un artista, ma anche un professionista, al quale il duca poté affidare la maggior parte delle sculture per la sua nuova dimora.

Tra queste è bene almeno ricordare: oltre a tre ritratti di Francesco Melzi d'Eril, i busti di Letizia Ramolino, Joséphine de Beauharnais e del figlio Eugenio, di Vittorio Alfieri e di Giocondo Albertolli, la statua di Cristo Redentore e il paliotto d'altare in altorilievo con la Sacra Famiglia, Sant'Elisabetta e San Giovannino nella cappella -oratorio e, ovviamente, il gruppo di Dante e Beatrice, l'opera più nota e di maggior impegno che ha lasciato dietro di sé più numerose tracce di storia e di critica.

Le circostanze della sua genesi si possono in gran parte ricostruire dalle lettere scritte dallo scultore e indirizzate a Francesco Melzi d'Eril, a Giocondo Albertolli, a segretari, collaboratori, amministratori, fornitori ed anche a colleghi artisti. Lettere che in varia misura, raccontano la storia del monumento, danno notizie sui luoghi, tempi e metodi di lavoro, a volte ne seguono il percorso e le trasformazioni dalle cave di marmo alla destinazione, giustificano scelte e rifacimenti ${ }^{28}$. Più in generale gettano luce sui rapporti tra artisti e committenti, su come, in un periodo come quello napoleonico, segnato da rapidi rivolgimenti politici e militari, gli eventi della grande storia si siano incrociati con le vicende private e i destini dei singoli, alternando momenti di stabile successo ad altri di grande precarietà: consentono, in altre parole, di collocare le opere d'arte nel concreto della vita delle persone.

Emerge innanzi tutto da parte di Comolli un forte sentimento di rispetto, di riconoscenza, ed anche di non disinteressata sottomissione nei confronti del duca. Gli si rivolge chiamandolo «Eccellenza», «dolce mecenate», «protettore», «benefattore» e «venerato padrone»,

28 Ringrazio il proprietario Fulco Gallarati Scotti per avermi consentito di studiare alcuni documenti privati di famiglia, d'ora in poi indicati con Documenti Villa Melzi (DVM). 
si firma sempre «um.mo, dev.mo, obbl.mo servitore», ma al di là delle formule di rito, queste espressioni indicano anche come per l'esercizio della scultura, bisognosa di materiale pregiato, di collaboratori, di trasporti onerosi, di spazi e attrezzature costose, con tempi di realizzazione mediamente lunghi, il favore dei grandi e dei potenti fosse condizione essenziale.

E' una situazione apertamente accettata, in un gioco di ruoli di cui entrambe le parti sono perfettamente consapevoli: Comolli sa che per chi si dedica «all'arte d'animare i sassi» ${ }^{29}$, è necessaria la «buona fortuna», fa voti per la salute del duca, dichiarando che da questa e dalla generale prosperità del suo mecenate sarebbero dipesi «il bene della Patria [...] la gloria d'Italia», e «la sicurezza della mia quiete»; chiede spesso aiuti finanziari al duca per disporre di quella tranquillità che gli avrebbe permesso di meglio servirlo. Da parte sua Melzi acconsente spesso di buon grado alle richieste, scrivendo, con squisita signorilità, che la tranquillità d'animo è condizione necessaria «ai lavori del genio» ${ }^{30}$.

Tornando tuttavia al monumento: tra il 1807 e il 1808 si ha notizia che lo scultore si era dedicato, con l'aiuto di esperti, alla ricerca e all'acquisto del marmo nelle cave delle Alpi Apuane del Ducato di Massa e Carrara annesso al Principato di Lucca e Piombino, retto da Elisa Bonaparte Baciocchi. Nel giugno del 1808 egli inviò a Melzi d'Eril la perizia di un marmo giudicato «il più bel pezzo statuario che siavi in Carrara», degno di «un grande mecenate» quale egli era ${ }^{31}$; nei mesi seguenti il blocco fu «strascinato» a Carrara per essere dirozzato nel laboratorio aperto appositamente in città dallo scultore, in ottemperanza alle norme stabilite dalla Banca Elisiana.

Fondata nel 1807 da Elisa Baciocchi per sostenere l'economia locale, la Banca caricava infatti di dazi diversi a seconda del valore, l'uscita dei marmi grezzi dal Principato, favorendo, di conseguenza, ogni opera lavorata sul posto, così da incoraggiare la presenza degli scultori,

29 L'espressione si trova in DVM, Lettera di G.B. Comolli a F. Melzi, Genova, 25 novembre 1806.

30 Citazioni da: DVM, Lettera di G.B. Comolli a F. Melzi, Carrara, 13 maggio 1811; e Lettera di F. Melzi a G.B. Comolli, Milano, 11 maggio 1809.

31 DVM, «Perizia della qualità del marmo» sottoscritta da Giovanni Battista Perugini e Paolo Bargigli architetto, 28 maggio 1808. 
l'apertura di laboratori, la produzione artistica nei luoghi della materia prima e l'impiego della mano d'opera locale specializzata ${ }^{32}$. In appoggio a tali provvisioni Francesco Melzi d'Eril fece quindi eseguire a Carrara la maggior parte delle opere in marmo destinate alla villa, mantenendovi Comolli con il suo atelier a periodi alterni per circa due anni ${ }^{33}$.

Dalla corrispondenza si evince che la scelta del tema si doveva allo stesso Melzi d'Eril e risaliva al 1806, di poco posteriore all'istituzione del Regno d'Italia ${ }^{34}$, ma antecedente il progetto della villa di Bellagio. Destinato in origine alla residenza milanese del duca, il soggetto si era andato poi precisando nel pensiero di rappresentare l'incontro fra Dante e Beatrice nel Canto XVIII del Paradiso, quando la donna amata consola il poeta della profezia di Cacciaguida sull'esilio indicandogli la superiore giustizia divina.

Se dal punto di vista artistico l'opzione dantesca non era forse estranea all'interesse coltivato, ad esempio, da Giuseppe Bossi, particolarmente vicino a Melzi d'Eril e tra gli autori del programma iconografico-decorativo della villa $a^{35}$, dal punto di vista ideologico-politico, poteva essere influente il pensiero di Vittorio Alfieri suo sincero e rispettato amico fin dagli anni Ottanta del Settecento, quando insieme avevano trascorso «un intiero inverno» a Parigi e, nonostante la diversità di carattere, avevano maturato una profonda concordanza intellettuale ${ }^{36}$. La Vita di Alfieri testimoniava del resto come già tra Sette e Ottocento il poeta fosse per lui non solo maestro di lingua e di stile ma anche di pensiero e di libertà, nonché portatore di una precoce coscienza del problema dell'identità nazionale: un modello ideale di arte ed etica, non estraneo alla consapevolezza della condizione di sot-

32 Cfr. Carozzi 1984, 559-581; Carozzi 1986, 437-450.

33 Marmottan 1901, 99.

34 Lo ricorda Comolli nel 1810: cfr. DVM, Lettera di G.B. Comolli a F. Melzi, Carrara, 8 luglio 1810.

35 Giuseppe Bossi (1777-1815) fu studioso di Dante, collezionista di pregiate edizioni delle sue opere, autore di disegni ispirati alla Commedia. Per la sua figura nel periodo in esame, cfr. Nenci 2004.

36 La testimonianza è di Bossi e si riferisce probabilmente all'inverno 1786-178: «Melzi passò un intiero inverno con Alfieri a Parigi. [...] Alfieri era impetuoso, iracondo, ardente, ma tenero. Melzi ed egli prendevano una loggia da soli per sentire le tragedie francesi. Alfieri studiava il suo mestiere. Melzi studiava Alfieri amando di vedere l'effetto che in tal uomo producevano quelle rappresentazioni». Cfr. Memorie inedite $1878,281-282$. 
tomissione italiana al dominio straniero ${ }^{37}$. Difficile quindi non percepire un significato o per lo meno un accenno di rivendicazione politica anche nella scelta artistica di Melzi d'Eril le cui aspirazioni per un paese indipendente e padrone del proprio destino erano state fortemente frustrate dal corso dell'Impero e del Regno d'Italia.

Per un soggetto così impegnativo lo sforzo di Comolli dovette essere notevole, nel tentativo di accordare le forme della scultura al testo della Commedia e far sì che arte e letteratura si sostenessero e chiarissero vicendevolmente. Vale la pena riportare alcuni passi della descrizione finale inviata al duca l'8 luglio 1810 (Fig. 7):

«Il soggetto proposto dall'E.V. è rappresentato nel Canto XVIII del Paradiso nel momento che il Poeta vien introdotto nell' [sic] Pianeta di Giove, ove la Sua Donna li fa vedere le anime di coloro che bene avevano amministrata giustizia al mondo. Dante è nell'atto d'andare rivolgendosi all'amoroso Suono del Suo Conforto, porge a Beatrice la sinistra mano, con l'altra porta il volume della Sua Divina Commedia, il capo è ornato d'alloro, è vestita la figura con l'abbito [sic] del suo secolo. Beatrice signoreggia il Sesto Pianeta, attraversato dal [sic] Zodiaco; lo tocca appena con la punta di piedi, atteggiata in questo modo per farle /se potevo/ prendere l'aspetto che le dà il suo autore quando dice sovra candido vel cinta d'olivo Donna m'apparve. E' scolpita nel momento preciso ch'Ella dice a Dante Ecco colui c'ogni torto disgrava. Posteriormente al gruppo v'è una grand'aquila che il Poeta introduce nell' [sic] medesimo pianeta, [...] rappresentata come la descrive l'autore Parea dinanzi a me con le ali aperte la Bella Imago. Ella è in atto di parlare non vedendo dall'aspetto principale altro di lei che quello che il Poeta prescrive. La testa e il collo d'un aquila vidi. Il Pianeta è circondato da nubi, ornato della Croce, per vedere con questo segno le parole di Dante io vidi per la Croce un

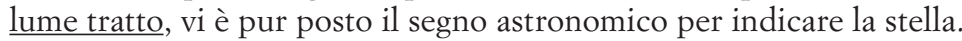
$[\ldots] \gg^{38}$.

37 Cfr. le considerazioni di Cedrati 2014. Cfr. inoltre A. Accame Bobbio 1970, ad vocem. Da ultimo, più in generale, Conti 2021.

38 DVM, Lettera di G.B. Comolli a F. Melzi, Carrara, 8 luglio 1810. 


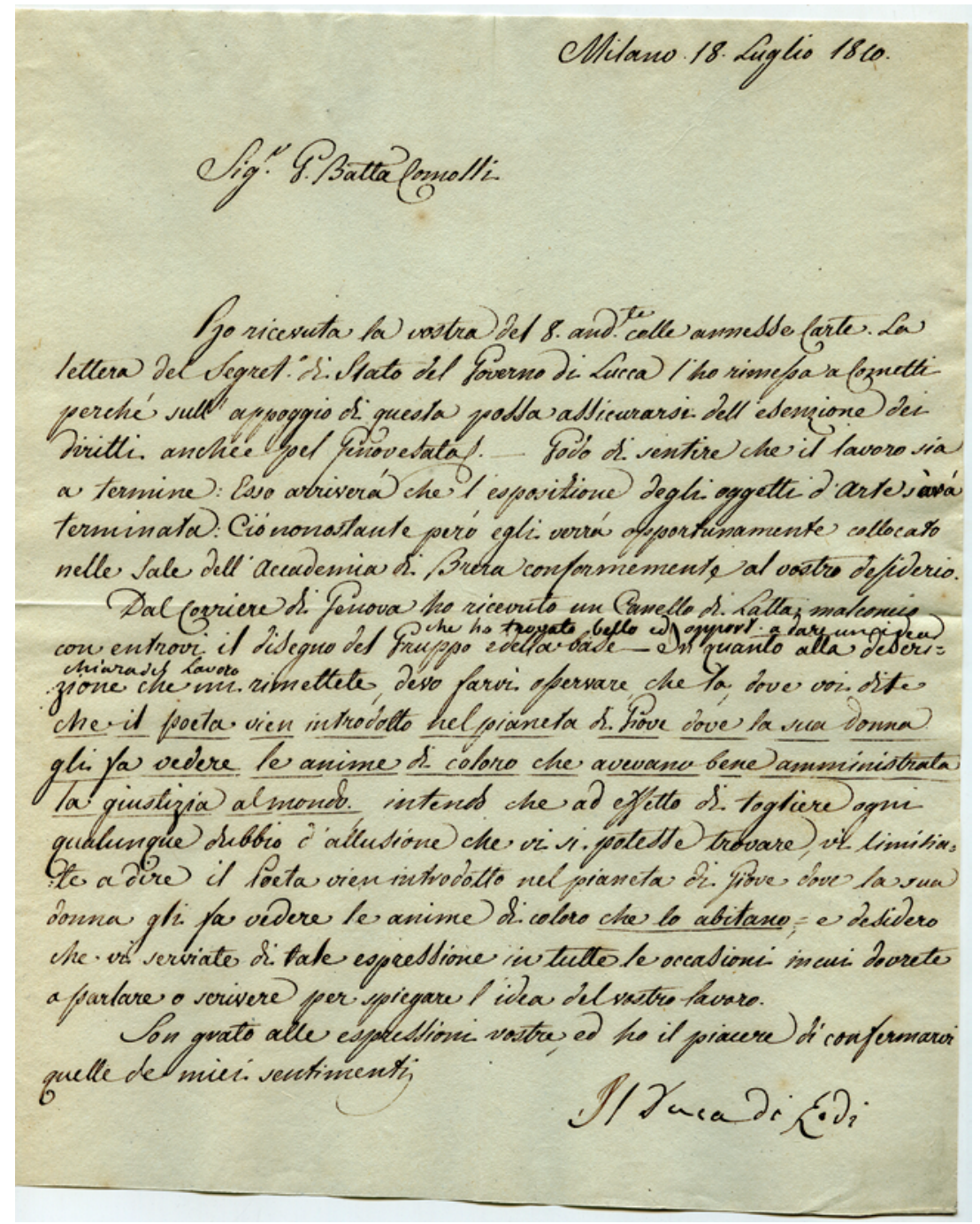

Fig. 7 - Lettera del Duca di Lodi Francesco Melzi d'Eril a Giovanni Battista Comolli in merito al monumento a Dante e Beatrice.

Milano, 18 luglio 1810. DVM. 
Il ruolo pacificatore di Beatrice, il senso di conforto che promana dalla sua persona nella consapevolezza che ogni umana miseria è spazzata via dalla superiore giustizia divina, è poi esaltata dai versi, incisi sul basamento che non reca nessun'altra iscrizione: «E quella Donna che a Dio mi menava/ disse muta pensier, pensa ch'io sono/ Presso a colui, ch'ogni torto disgrava/ Io mi rivolsi all'amoroso Suono/ del mio Conforto» ${ }^{39}$.

Di quest'opera lo scultore si dimostrava sia soddisfatto sia timoroso, nella consapevolezza che le difficoltà della realizzazione, l'impegno interpretativo, il tema stesso rappresentavano una novità nel panorama artistico dell'epoca e una precisa responsabilità nei confronti del committente. E' significativo infatti che, dopo avere ricevuto la descrizione, quest'ultimo raccomandasse di evitare ogni possibile allusione alla sua persona chiedendo a Comolli di attenersi a spiegazioni più "neutre":

«Devo farvi osservare che là dove voi dite che il poeta vien introdotto nel pianeta di Giove dove la sua donna gli fa vedere le anime di coloro che avevano bene amministrato la giustizia al mondo, intendo che» al fine di «togliere ogni qualunque dubbio d'allusione che vi si potesse trovare, vi limitate a dire il Poeta vien introdotto nel pianeta di Giove dove la sua donna gli fa vedere le anime di coloro che lo abitano, e desidero che vi serviate di tale espressione in tutte le occasioni in cui dovrete o parlare o scrivere per spiegare l'idea del vostro lavoro». ${ }^{40}$

I contenuti artistici e il prestigio del duca richiamarono l'attenzione del pubblico e la sostanziale approvazione dell'opera mentre era ancora in lavorazione. Lo testimonia tra gli altri, nel 1810, Giorgio Grognet, architetto e «Ajutante del Genio Imperiale» sul «Giornale Enciclopedico di Firenze» dove si legge di una Carrara che, come «l'isola di Paros [...] ai fortunati tempi di Pericle», raccoglieva i migliori scultori per lavorare i «suoi candidissimi marmi $»^{41}$. «Gran cose poi dovrei dirvi del merito del Signor Comolli», proseguiva l'autore, dichiarando che per mancanza di spazio doveva limitarsi a segnalare come nel suo studio» si trovasse una collezione di «oggetti finiti, o quasi finiti con arte somma, destinati tutti per il Signore Principe Melzi di Milano, anzi

39 DVM, Lettera di G.B. Comolli a F. Melzi, Genova, 25 novembre 1806.

40 DVM, Lettera di F .Melzi a G.B. Comolli, Milano, 18 luglio 1810. Sottolineature in originale.

41 Grognet 1810, 98. 
da lui commissionati $[\ldots]$.». Tra questi era già celebre e «andava per le bocche di tutta quasi l'Italia», il gruppo di Dante «condotto al cielo dalla sua Beatrice» ${ }^{42}$ (Fig. 8).

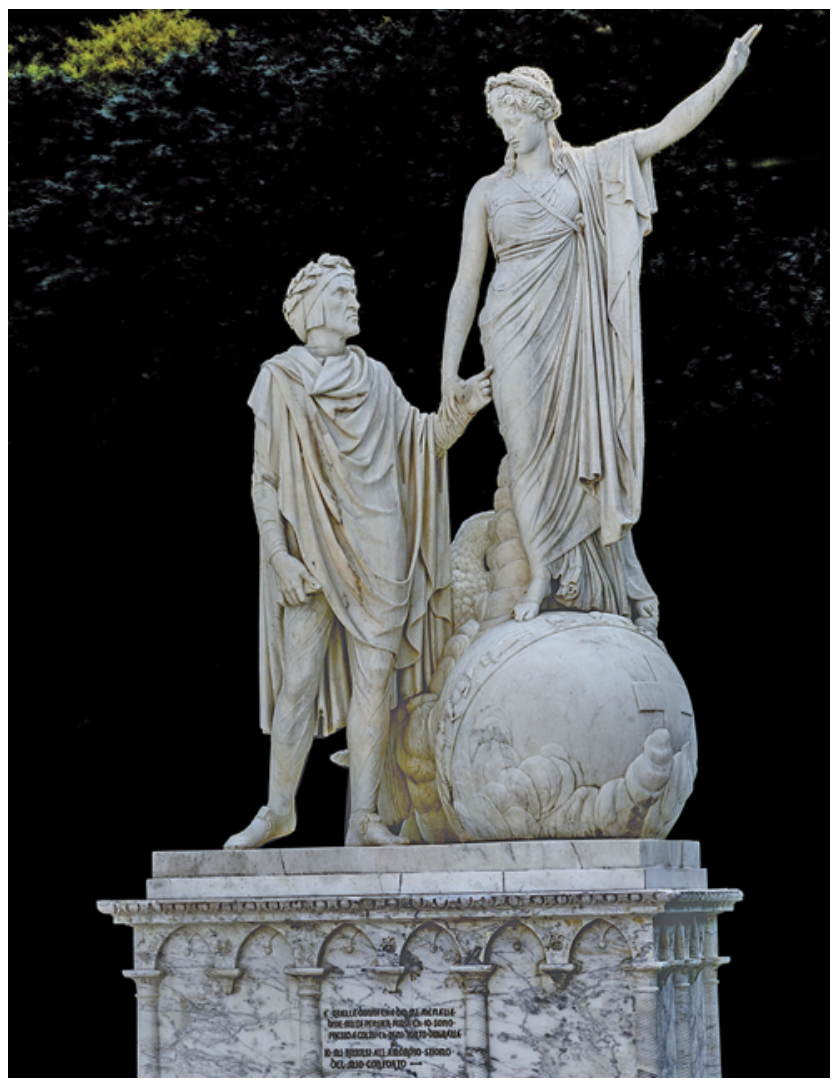

Fig. 8 - Veduta del monumento a Dante e Beatrice a Bellagio. Foto: Franco Papetti.

La scultura suscitava curiosità e interesse anche per l'impronta stilistica che la differenziava dalla produzione del periodo, testimoniando di orientamenti "moderni", oltre il neoclassicismo e in anticipazione di possibili ideali romantici. A proposito del basamento, disegnato con la super-

42 Grognet 1810, 99-100. 
visione di Comolli dall'architetto Paolo Bargigli ${ }^{43}$, si sottolineava, ad esempio, la foggia a guisa di «sarcofago antico, ma di antichità gotica, e non già greco-romana»; si evidenziavano i caratteri gotici dell'iscrizione incisi nel marmo e riempiti di rosso, «alludenti all'Epoca di quel Padre della lingua più bella», mentre la sequenza di archetti ogivali nella parte superiore della «magnifica base» rendeva immediatamente riconoscibile il richiamo al Medio Evo, alla sua arte e ai tempi del poeta ${ }^{44}$.

Si può osservare che le figure a grandezza naturale si presentano nitide ed estatiche, ma, nel caso di Dante, già animate dal desiderio di un parziale realismo: inusualmente ritratto in abiti medievali, il poeta abbandonava infatti la dimensione ideale a favore della fedeltà storica, così come allora si poteva intendere. Comolli, sempre attento alla fisionomia dei personaggi, dovette certo arrovellarsi sui lineamenti e l'atteggiamento del poeta, tanto da rivedere più volte il bozzetto e da verificarne l'adeguatezza in base ai modelli di cui veniva a conoscenza. Nell'aprile del 1809 scriveva di avere preso la decisione di «rinnovare del tutto la figura di Dante», nonostante «l' immensa fatica» che comportava il cambiamento e «dovendo [...] stare nelle dimensioni del sasso di già quasi abbozzato», convinto tuttavia di ottenere un risultato migliore, «che non dispiacerà a buoni e forse farà tacere i malevoli» ${ }^{45}$.

«Malevoli» o meno, non tutti taceranno proprio riguardo alla figura di Dante, considerata nell'insieme più incerta e meno riuscita di quella di Beatrice, nel tentativo, certo non facile, di armonizzare la figurazione realistica con quella idealizzata, il personaggio storico con la sua immagine culturale. Il giudizio su Dante, non aiutato per altro dalle fattezze poco armoniose del volto, è stato effettivamente severo a fronte di quello molto più favorevole su Beatrice ${ }^{46}$. Per lo stesso Comolli, la scultura sembrava a volte identificarsi soprattutto con la grazia di quest'ultima, tanto da riferirsi sovente al «gruppo di Beatrice» o addirittura a «Beatrice».

43 Con Paolo Bargigli (1760-1815) architetto livornese, fervente filofrancese, attivo anche a Milano nel primo periodo della dominazione francese, Comolli aveva collaborato in più occasioni, condividendone senza riserve la fede politica. Cfr. Ferretti 2005; Grassi 2007, 387-390.

44 Grognet 1810, 100.

45 DVM, Lettera di G.B. Comolli a F. Melzi, Carrara, 26 aprile 1809.

46 Cfr., a titolo di esempio, Villa Melzi 1907, 38: «La figura di Dante sente un po' del tozzo; ma quella di Beatrice è piena di voluttà, di affetto e di celestiale sicurezza». 
Nell'agosto del 1810 il monumento era ultimato e Comolli lo accompagnò nel viaggio a Milano: via terra da Carrara a La Spezia, e poi via mare facendo vela fino a Genova, in un percorso che tenne in ansia lo scultore, attanagliato da «penosa smania per tema di perdere Beatrice» all'incontro coi «legni nemici», vale a dire coi brigantini inglesi fermi in agguato al largo di Livorno e pronti a razziare i vascelli carichi di marmi ${ }^{47}$. Il viaggio non fu facile anche nei tratti seguenti e, in particolare, nell'attraversamento del Po che non poté effettuarsi sui ponti galleggianti ormai «sdruciti» per i troppi passaggi degli eserciti; furono necessari quindi un barcone e un carretto speciale del tipo usato per il trasporto dei cannoni ${ }^{48}$. Non sfuggirà l'interesse di queste annotazioni, che aprono squarci non soltanto sulla cultura materiale che sta dietro alle opere d'arte, ma anche sulla geografia politica e militare dell'Italia napoleonica, sui suoi paesaggi e sulla loro organizzazione territoriale.

Arrivato a Milano all'inizio di ottobre 1810, il gruppo venne esposto il mese seguente «sulla piazza di Brera» assecondando il desiderio dello scultore e dello stesso Melzi di mostrarlo in ambiente accademico. Come già sottolineato, era stato quest'ultimo a proporre il soggetto nell'auspicio di un'opera che onorasse Dante e "desse corpo" al testo poetico di un Canto come il XVIII del Paradiso, particolarmente complesso e denso di sequenze narrative, richiami e prospezioni.

Dal punto di vista della rappresentazione artistica esso offriva la possibilità di raffigurare insieme, in atteggiamento calmo e plasticamente equilibrato, i due protagonisti della Commedia; mentre dal punto di vista dei contenuti letterari il Canto, consentiva, pur rimanendo sottotraccia, il compiacimento di legare l'arte e la storia all'attualità. In un certo senso intensificava l'omaggio al poeta nel considerarlo "dentro" alla sua opera in una situazione intrisa di significati morali e religiosi, ma pur sempre legata agli eventi dell'esistenza terrena, non lontana da

47 DVM, Lettera di G.B. Comolli a F. Melzi, Carrara, 31 agosto 1810: «Dopo una penosa smania per tema di perdere Beatrice, sono arrivato in questo porto alle otto di questa mane. Gli Inglesi sono immobili [...] quasi sulla costa da Livorno a Genova, ed io sono stato refuggiato [sic] al Golfo qualche giorno per non essere loro preda, a attendere che avessero la compiacenza di prendere il largo per approfittare della circostanza, come feci avanti jeri non senza palpiti, ed arrivai felicemente».

48 DVM, Lettera del consigliere L. Bossi a F. Melzi, Milano, 2 ottobre 1810 dove si ragguaglia il duca sul "viaggio" del monumento da Carrara a Milano. 
quell'intreccio tra la poesia e la vita che ha costituito caratteristica ineludibile della sua arte ${ }^{49}$.

Le figure emblematiche che si affacciano nel Canto sono numerose e pregnanti: nel Cielo di Marte sono il globo terrestre, i segni zodiacali, la croce formata dalle anime dei Combattenti per la fede; nel Cielo di Giove sono le anime degli spiriti giusti unite a formare lettere dell'alfabeto simili a uccelli che, dopo essersi levati in volo, formano schiere di diversa forma. La «M gotica» è l'ultima lettera che si rivela e che dà origine a una decisiva metamorfosi: la figura di un giglio araldico e, successivamente, quella di un'aquila; vale a dire l'aquila imperiale che, nella prospettiva dantesca, è protagonista della storia in quanto riflesso del volere divino, simbolo della storia millenaria dell'Impero e della sua insostituibile funzione ordinatrice ${ }^{50}$.

Alcuni di questi soggetti sono presenti nel monumento di Bellagio: Il globo crociato denso di nubi rappresentante il V Cielo del Paradiso (nel pianeta di Marte), la Croce per le anime dei Giusti (nel pianeta di Giove) e, soprattutto l'aquila imperiale ${ }^{51}$. Il suo disegno riprende l'insegna trionfale delle legioni romane, la stessa adottata da Napoleone nel 1804 come simbolo di un Impero che intendeva riallacciarsi alla gloria dell'antico. Aquile in bronzo dorato furono issate sulle aste delle bandiere e degli stendardi di tutti i reggimenti dell'esercito francese fino al 1815, distinguendosi per le ali spiegate, il becco aperto, la zampa posata sulla faretra di Giove, in conformità al famoso «modello 1804» ideato dallo scultore Antoine-Denis Chaudet e realizzato dal fonditore parigino Pierre-Philippe Thomire: un modello rigorosamente stabilito che, nel 1805, fu consegnato per le repliche ai reparti della Guardia Reale Italiana ${ }^{52}$ (Fig. 9).

Non esiste emblema che sia del tutto innocuo e, ovviamente, l'aquila napoleonica non fa eccezione. Il modello originale, del 1804, presenta il capo girato a est, ovvero guarda dalla Francia verso l'Europa. E', in un

49 Cfr. le considerazioni di Spera 2008, 13-19. E' opportuno ricordare che l'autore della Vita di Dante nel 1921, fu il letterato, intellettuale e diplomatico, Tommaso Gallarati Scotti (1878-1966), discendente di Francesco Melzi d'Eril e allora proprietario della villa di Bellagio.

50 Per i riferimenti alla Divina Commedia e in particolare al Canto XVIII del Paradiso ho utilizzato il sito Paradiso Canto XVIII - La Divina Commedia (weebly.com).

51 DVM, Lettera di F. Melzi a G.B. Comolli, Milano, 18 luglio 1810,

52 Cfr. Bascapè - Del Piazzo 1983, 750-770. 
certo senso, l'aquila dell'impero francese che scruta il suo terreno di conquista dove è compresa anche l'Italia, il suo più stretto alleato. Un atteggiamento non propriamente rispettoso e forse non molto apprezzato, tanto che nel 1805, per le insegne dei reggimenti italiani si pensò a aquile identiche, ma con il capo rivolto ad ovest, cioè dall'Italia alla Francia nel segno di reciproche alleanze più che di sudditanze. Nella realtà furono pochissime le aquile italiane realizzate in questo modo, per scelta politica, oltre che per semplificazione logistica ed economica, così che la maggior parte dei reggimenti italiani dovette adottare il modello francese ${ }^{53}$.

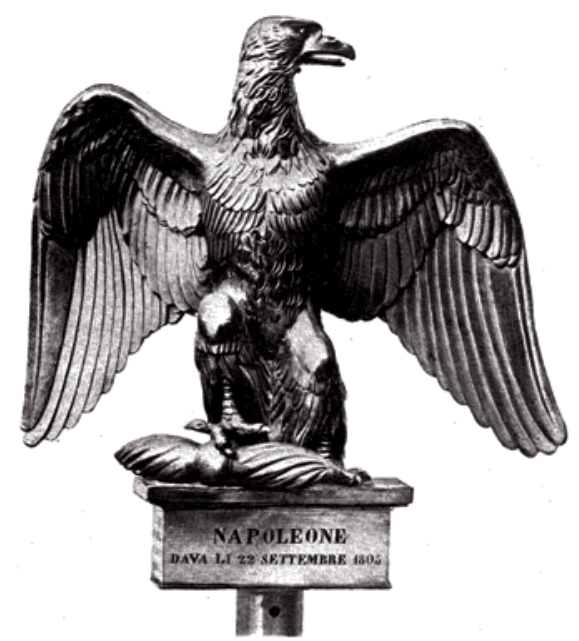

Fig. 9 - Disegno per l'aquila napoleonica da apporre sull'asta delle bandiere a insegna dell'Esercito del Regno Italico 1805-1814.

Da: Insegne e simboli. Araldica pubblica e privata medievale e moderna,

A cura di G.C. Bascapè, M. Del Piazzo, Roma, Ministero per i beni Culturali e Ambientali, 1983.

Osservando il monumento di Bellagio si capisce quindi come non sia irrilevante l'adozione di questa particolare aquila collocata dietro le figure di Dante e Beatrice: significativamente essa guarda ad ovest, ovvero dall'Italia alla Francia. Siamo sì davanti a un'aquila uguale a quelle

53 Ringrazio per queste precisazioni l'architetto Piersergio Allevi, esperto di armi e uniformi antiche, il cui aiuto è stato determinante nella lettura del monumento e dei suoi riferimenti napoleonici. 
napoleoniche, ma soprattutto siamo davanti a un'aquila italiana che anche così, nella direzione e nella fierezza dello sguardo, rafforza l'omaggio al grande poeta e a un pensiero di patria che dalla sua opera si poteva irraggiare sul luogo e sul committente (Fig. 10). Non a caso Felice Calvi, ne Il Patriziato Milanese scriverà che alcuni «alcuni grandi uomini, sorvolano come aquile alla folla dei mortali e fanno onore a tutta una città», citando fra questi Ansperto, Ariberto da Intimiamo, San Carlo, Federico Borromeo, Cesare Beccaria, e, per l'appunto, Francesco Melzi d'Eril ${ }^{54}$.

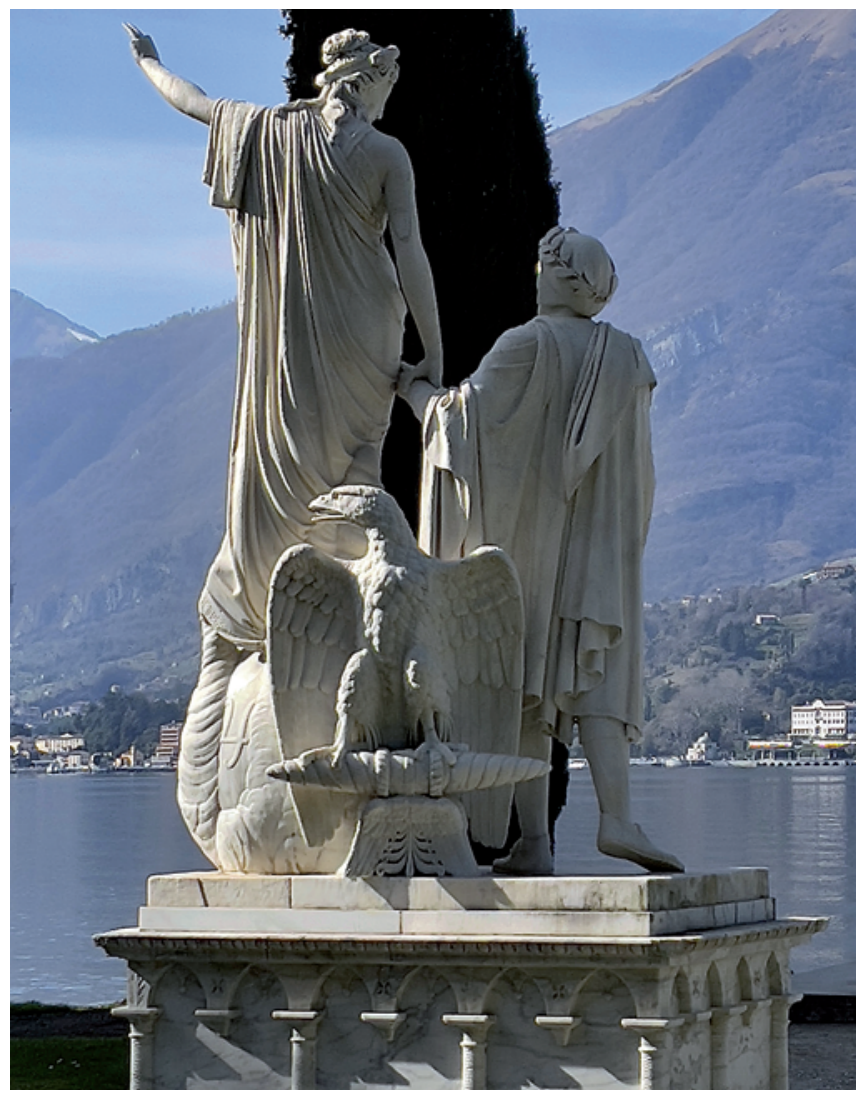

Fig. 10 - Il fronte posteriore del monumento a Dante e Beatrice con "l'aquila italiana". Foto: Franco Papetti.

54 Calvi 1866, 177. 
Dopo l'esposizione a Milano e forse dopo un breve "soggiorno" nel giardino di palazzo Melzi in città, il monumento a Dante e Beatrice fu trasportato a Bellagio e collocato definitivamente, tra il 1813 e il 1814, nel giardino della villa in quella che è ancor oggi la sua posizio$n e^{55}$. Non vi sono ad ora di notizie specifiche sulla data esatta e sulle ragioni di questa decisione, ma si può pensare che, oltre al desiderio di preservarlo in luogo più tranquillo, contasse ormai per il duca l'idea di fare di Bellagio non soltanto un soggiorno ameno e confortevole, ma anche un luogo dove raccogliere le memorie, coltivare le inclinazioni di gusto e asserire i propri ideali.

Egli non volle essere considerato tra i giusti, ma, almeno in parte, dovette considerarsi vittima di un'ingiustizia. Certamente non fu costretto all'esilio, ma, in un certo senso, visse il senso della perdita e dell'estraneità che questo comporta, quando il venire meno delle sue aspirazioni lo allontanò sempre di più dal sentimento della politica, rendendo nel contempo Bellagio un luogo di possibile "risarcimento": un suo privato Paradiso dove era "giusto" che il monumento si trovasse.

La morte nel gennaio del 1816 lasciò a Francesco Melzi d'Eril ben poco tempo per poter apprezzare il suo «buen ritiro», ma la scultura di Dante e Beatrice divenne presto un landmark, e una tra le mete preferite dei visitatori che, nel corso del tempo, hanno percorso i giardini di Bellagio. Nel Viaggio al lago di Como di Davide Bertolotti, una delle più diffuse guide di viaggio del primo Ottocento, si legge che il duca «amava la grandezza dell'Italia» ${ }^{56}$, il che può essere una sintetica ma efficace chiave di lettura per un monumento dove la figura di Dante, per la sua opera, la scelta linguistica, la fede politica e le circostanze della vita: in altri termini per il suo essere profondamente calato nell'arte e nella storia d'Italia, assumeva il ruolo di exemplum della cultura e identità nazionale.

Virtù, queste, non estranee a villa Melzi d'Eril e che, seppure in chiave antifrancese, furono riconosciute anche in piena Restaurazione; quando, ad esempio, nel 1838, l'imperatore Ferdinando I vi soggiornò durante il viaggio che dall'Austria lo avrebbe portato a Milano per esse-

55 Per l'arrivo a Bellagio cfr. DVM, Nota di G. Albertolli al duca, 11 maggio 1814. Il documento riporta i pagamenti per l'imballaggio delle sculture, ma non si riferisce a una data precisa del loro arrivo sul lago.

56 Bortolotti 1821, p.81. 
re incoronato Re del Lombardo Veneto. Nei resoconti dell'epoca si legge che la meta era stata scelta per «l'amenità della postura» e per essere «un tempio di italianità» ${ }^{57}$, rivelandoci un apprezzamento non scontato in quel momento storico, a proposito di un luogo che aveva avuto origine nell'Italia napoleonica ed era la creazione di Francesco Melzi d'Eril dai chiari trascorsi antiaustriaci.

$\mathrm{Ma}$, evidentemente, contava il senso complessivo e la percezione di un contesto che, per la qualità naturale e la cura con cui era stato allestito, appariva fortemente ispirato al sentimento del paese: anche attraverso il monumento dedicato a Dante e a Beatrice nei giardini e nel paesaggio del lago (Fig. 11).

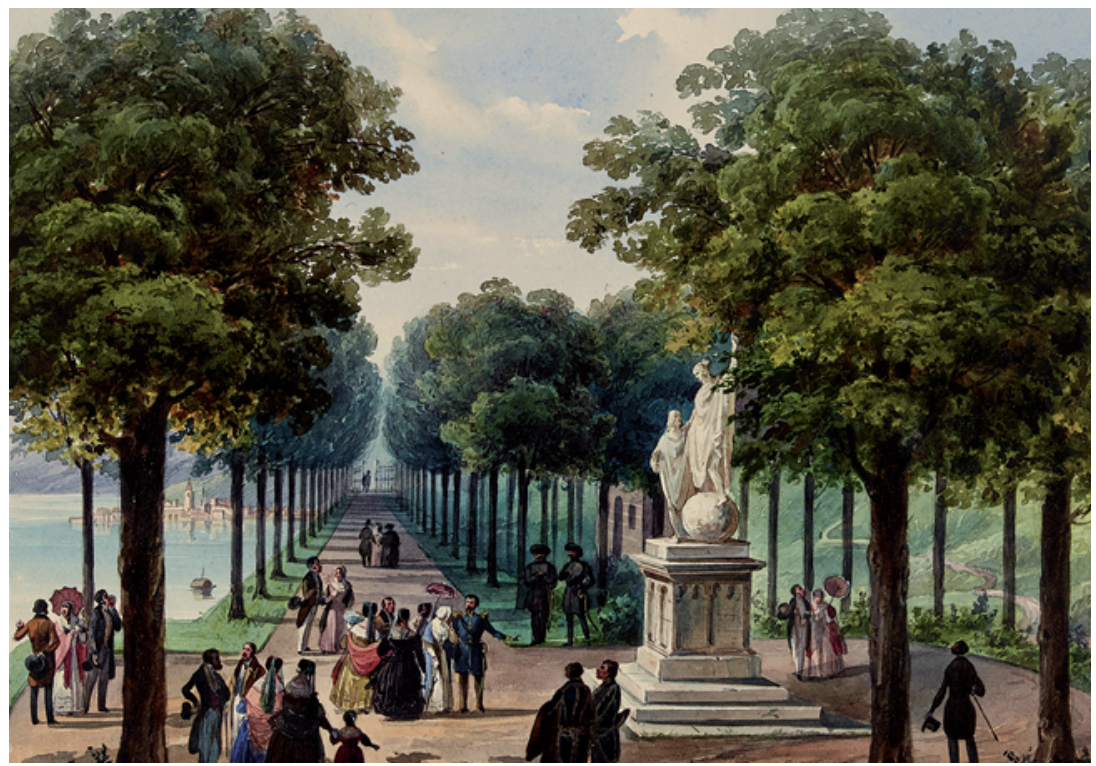

Fig. 11 - Il monumento a Dante e Beatrice nei giardini di Bellagio in un acquerello di Alessandro Sanquirico, s.d. [post 1842]. Bellagio, Museo Lodovico Gallarati Scotti.

57 Itinerario del viaggio 1838; DVM, «Descrizione della festa a Villa Melzi per il soggiorno delle Maestà Imperiali Ferdinando I», s.d. [ma agosto 1838]. 


\section{BIBLIOGRAFIA}

Accame Bobbio, Aurelia 1970 - Alfieri Vitttorio, in Enciclopedia dantesca, Roma, Treccani 1970, vol. I.

Agliati - Cordera - Ricci (a cura di) 2019 - Ornato e architettura nell'Italia neoclassica. Il fondo degli Albertolli di Bedano, secc. XVIII-XIX, Bellinzona, Edizioni dello Stato del Cantone Ticino.

Bascapè, Giacomo C. - Del Piazzo. Marcello (a cura di) 1983 - Araldica napoleonica in Italia, in Insegne e simboli. Araldica pubblica e privata medievale e moderna, a cura di G.C. Bascapè, M. Del Piazzo con L. Borgia, Roma. Ministero per i beni Culturali e Ambientali.

Bortolotti, Davide 1821 - Viaggio al lago di Como, Como, Ostinelli.

Brambilla, Elena - Capra, Carlo - Scotti, Aurora (a cura di) 2008. Istituzioni e cultura in età napoleonica, Milano, FrancoAngeli.

Busto del Vice-Presidente 1804. Busto del Vice-Presidente Melzi, in «Nuovo Giornale dei Letterati», tomo I, II semestre, 1804.

Calvi, Felice 1866 - Il patriziato milanese, secondo nuovi documenti deposti negli archivi pubblici e privati, Milano, Andrea Mosconi.

Cantù, Cesare 1847 - Guida al lago di Como ed alle Strade di Stelvio e Spluga, Como, Ostinelli (I ediz. 1831).

Capra, Carlo - Della Peruta, Franco - Mazzocca, Fernando 2002. Napoleone e la Repubblica Italiana: 1802-1805, Milano Skira.

Capra, Carlo 2009 - Melzi d'Eril Francesco, in Dizionario biografico degli italiani, Roma, Treccani, vol. LXXIII.

Carozzi, Renato 1984 - La Banca Elisiana, in Il principato napoleonico dei Baciocchi, Lucca, Nuova Grafica Lucchese.

Carozzi, Renato 1986 - Artisti e artigiani sotto il segno dell'Impero a Carrara, in Il principato napoleonico dei Baciocchi (1805-1814). Riforma dello stato e società, Atti del convegno internazionale, a cura di V. Tirelli, Lucca, Banca del Monte di Lucca, Pacini Fazzi.

Cedrati, Chiara 2014 - La liberà dello scrivere. Ricerche su Vittorio Alfieri, Milano, Edizioni Universitarie di lettere, Economia, Diritto.

Colle, Enrico - Mazzocca, Fernando 2005 - Il trionfo dell'ornato, Giocondo Albertolli (1742-1839), Cinisello Balsamo, Silvana Editoriale.

Conti, Fulvio 2021 - Il Sommo Italiano. Dante e l'identità della nazione, Roma, Carocci

D’Adda, Roberta - Onger, Sergio (a cura di) 2021. Dante e Napoleone. Miti fondativi nella cultura bresciana di primo Ottocento, Milano, Skira, Fondazione Brescia Musei.

Del Bianco, Nino 2002 - Nino del Bianco, Francesco Melzi D'Eril. La grande occasione perduta, Milano, Corbaccio.

Della Peruta, Franco 2007 - Cultura e organizzazione del sapere nella Lombardia dell'Ottocento. L'Istituto Lombardo di Scienze e Lettere dalla fondazione all'unità d'Italia, in L'Istituto Lombardo Accademia di Scienze e Lettere, vol. I, Storia Istituzionale, a cura di A. Robbiati Bianchi, Milano, Istituto Lombardo Accademia di Scienze e Lettere, Libri Scheiwiller. 
Ferretti, Mario 2005 - Paolo Bargigli e Vincenzo Revelli all'Elba. Due artisti giacobini al seguito di Napoleone, Ospedaletto, Pacini Editore.

Festa per l'Abbruciamento 1798. Festa per l'Abbruciamento del Libro d'Oro Processi del S. Ufficio e de' Patriotti [sic] progettata dal cittadino Pietro Guerrini membro dell'alta Pretura, ed eseguita dal cittadino Paolo Bargigli Architetto e dal cittadino Gio. Battista Comolli Scultore, Roma, Puccinelli.

Giambattista Comolli 1831. Giambattista Comolli, «Biblioteca italiana», vol. 61, gennaio. Grandesso, Stefano 2013 - Comolli e la scultura monumentale: l'allegoria politica nella scultura in Italia tra Impero e Restaurazione, «I Quaderni di Villa Monastero», Cattaneo, Oggiono.

Grano, Leonardo (a cura di) 1990 - G. B. Comolli scultore valenzano: l'uomo e l'artista, a cura di L. Grano, Valenza, Battezzati.

Grassi, Rosella 2007 - Paolo Bargigli, in Contro il barocco. Apprendistato a Roma e pratica dell'architettura civile in Italia 1780-1820, a cura di A. Cipriani, G.P. Consoli, S. Pasquali, Roma, Campisano Editore.

Grognet, Giorgio 1810 - N.16. Lettera di Giorgio Grognet Ajutante del Genio Imperiale ed Architetto, al Signor D. Marco Mastrofini Institutore di Filosofia nel Seminario di Frascati, nella quale gli dà ragguaglio di una sua gita a Carrara. Dal Golfo della Spezia li 18 Aprile 1810, «Giornale Enciclopedico di Firenze», tomo II, 1810.

Hubert, Gérard 1964 - La sculpture dans l'Italie Napoléonienne, Paris, De Boccard.

Itinerario del viaggio 1838. Itinerario del viaggio da Vienna a Milano e descrizione del Cerimoniale per il solenne ingresso in questa città dell'Augusto Imperatore Ferdinando I e di S.M. l'Imperatrice per l'incoronazione che avrà luogo nel giorno 6 settembre 1838 coll'indicazione delle giornaliere feste, Novara, Artaria.

Kannès, Gianluca 1982 - Comolli, Giovanni Battista, in Dizionario Biografico degli Italiani, Roma, Treccani, 1982, vol. XXVII.

Marmottan, Paul 1901 - Les arts en Toscane sous Napoléon. La Princesse Élisa, Paris, Honoré Champion.

Mazzocca, Fernando - Morandotti, Alessandro - Colle, Enrico 2001 - Milano neoclassica, Milano, Longanesi.

Mazzocca, Fernando 2021 - Napoleone e Milano tra realtà e mito. L'immagine di Napoleone da liberatore a imperatore, Milano, Skira.

Memorie inedite 1878. Memorie inedite di Giuseppe Bossi, «Archivio storico lombardo», $\mathrm{V}$, fasc. 2, giugno.

Nenci, Chiara 2004 - Le memorie di Giuseppe Bossi. Diario di un artista nella Milano napoleonica, Milano, Jaca Book.

Nicodemi, Giorgio 1915 - Lo scultore G.B. Comolli, «Vita d'arte», vol. 14, fasc. 87, marzo.

N. 276. Reale Lettera 1807. N. 276. Reale Lettera Patente che conferisce il titolo di duca di Lodi al signor Melzi cancelliere guardasigilli della Corona, «Bollettino delle Leggi del Regno d'Italia», parte III, 20 dicembre.

Pellissetti, Laura Sabrina 2009 - Villoresi Luigi, in V. Cazzato (a cura di), Atlante del giardino italiano, 1750-1940. Dizionario biografico di architetti, giardinieri, botanici, committenti, letterati e altri protagonisti, Roma, Istituto Poligrafico e Zecca dello Stato. 
Rosa, Marina (a cura di) 2009 - La Villa, i Giardini e il Parco di Monza nel fondo disegni delle Residenze Reali Lombarde, Milano, Skira.

Rosa, Marina 2014 - La Villa di Monza da Palazzo Nazionale a Villa Reale, in La Villa Reale di Monza dalla Repubblica Cisalpina al Primo Regno d'Italia", Viterbo, Betagamma Editore.

Selvafolta, Ornella 2010 - Lo scultore Giovanni Battista Comolli, Francesco Melzi e Giocondo Albertolli. Vicende artistiche di villa Melzi a Bellagio, «Archivio Storico Lombardo», serie XII, a. CXXXVI, vol. XV.

Selvafolta, Ornella 2012 - I giardini di villa Melzi d'Eril a Bellagio. Un museo all'aperto tra natura, arte e storia, Milano, Giardini di villa Melzi- Cisalpino, 2012.

Selvafolta, Ornella 2020 - Villa Melzi d'Eril a Bellagio: giardino e paesaggio sul lago di Como nella prima metà dell'Ottocento, in L'architettura del giardino in Europa. Evoluzione storica e nuove prospettive, a cura di F. Zecchino, Napoli, arte'm Prismi, Editrice Politecnica.

Silva, Ercole 1813 - Dell'arte de' giardini inglesi, Milano, Pietro e Giuseppe Vallardi, (I ediz. 1801).

Spera, Francesco 2008 - Introduzione alla Vita di Dante di Tommaso Gallarati Scotti, in Tommaso Gallarati Scotti, Vita di Dante a cura di F. Spera, Milano, FrancoAngeli.

Tedeschi, Letizia - Repishti, Francesco (a cura di) 2011 - Luigi Canonica. Architetto di utilità pubblica e privata, a cura di L. Tedeschi e F. Repishti, Cinisello Balsamo, Mendrisio Academy Press-Silvana Editoriale.

Tobia, Bruno 1997 - La statuaria dantesca nell'Italia liberale: tradizione, identità e culto nazionale, «Mélanges de l'École française de Rome. Italie et Méditerranée», (http://www.persee.fr/doc/mefr_1123-9891_1997_num_109_1_4479). Villa Melzi 1907 - Villa Melzi, in Ville e castelli d'Italia. Lombardia e laghi, Milano, Tecnografica. 